\title{
Energy and Flux Measurements of Ultra-High Energy Cosmic Rays Observed During the First ANITA Flight
}

H. Schoorlemmer*, K. Belov, A. Romero-Wolf, D. García-Fernández, V. Bugaev, S. A. Wissel, P. Allison, J. Alvarez-Muñiz, S. W. Barwick, J. J. Beatty, D. Z. Besson, W. R. Binns, W. R. Carvalho Jr., C. Chen, P. Chen, J. M. Clem, A. Connolly, P. F. Dowkontt, M. A. DuVernois, R. C. Field, D. Goldstein, P. W. Gorham, C. Hast, C. L. Heber, T. Huege, S. Hoover, M. H. Israel, A. Javaid, J. Kowalski, J. Lam, J. G. Learned, K. M. Liewer, J. T. Link, E. Lusczek, S. Matsuno, B. C. Mercurio, C. Miki, P. Miočinović, K. Mulrey, J. Nam, C. J. Naudet, J. Ng, R. J. Nichol, K. Palladino, B. F. Rauch, J. Roberts, K. Reil, B. Rotter, M. Rosen, L. Ruckman, D. Saltzberg, D. Seckel, D. Urdaneta, G. S. Varner, A. G. Vieregg, D. Walz, F. Wu, E. Zas

Full affiliation list can be found at

http://arxiv.org/abs/1506.05396

E-mail: harmscho@mpi-hd.mpg.de

\begin{abstract}
The first flight of the Antarctic Impulsive Transient Antenna (ANITA) experiment recorded 14 radio signals that were emitted by downward going cosmic-ray induced air showers. The dominant contribution to the radiation comes from the deflection of positrons and electrons in the geomagnetic field and is beamed in a cone around the direction of motion of the air shower. This radiation is reflected from the ice and subsequently detected by the ANITA experiment at a flight altitude of $\sim 36 \mathrm{~km}$. In this contribution, we estimate the cosmic-ray energy of the 14 individual events and find a mean energy of $2.9 \times 10^{18} \mathrm{eV}$. By simulating the ANITA flight, we calculate its exposure for ultra-high energy cosmic rays (UHECRs) and we estimate for the first time the cosmic-ray flux derived only from radio observations.
\end{abstract}

The 34th International Cosmic Ray Conference,

30 July- 6 August, 2015

The Hague, The Netherlands

\footnotetext{
* Speaker.
} 


\section{Introduction}

The ANITA-I experiment flew in the 2006-2007 Austral summer over Antarctica suspended from a high altitude long duration balloon. It was equipped with an array of 32 dual-polarized quad-ridged horn antennas which were optimized to observe radiation in the $200-1200 \mathrm{MHz}$ band. At its float altitude of $\sim 36 \mathrm{~km}$ it provided a panoramic view on the ice sheet below it. For more details about instrument functionality and performance see [1].

The goal of the ANITA-I experiment was to observe impulsive radiation from ultra-high-energy neutrino interactions in the ice. After careful rejection of man-made impulses, continuous waves, and thermal noise events, 16 impulsive events remained. However, their polarization signature was not what was expected from neutrino signals but in agreement with the expectation of radio signals from air showers [2]. Two of these signals were received directly from air showers that skimmed the Earth's atmosphere while the other 14 came from down-going air showers and their radiation was reflected off the ice before reaching the payload.

When these events were recorded, none of the available models for radio emission from air showers were able to predict observable signals in the $200-1200 \mathrm{MHz}$ frequency band. However, the recent inclusion of the effect of the atmospheric refractive index increased the frequency at which the simulations result in coherent radiation up to several $\mathrm{GHz}[3,4,5]$ and this provides now the opportunity to compare the observed signals directly to emission models. Figure 1 shows the footprint of the radio signal on the ground and indicates the parameters that are used to describe the geometry.

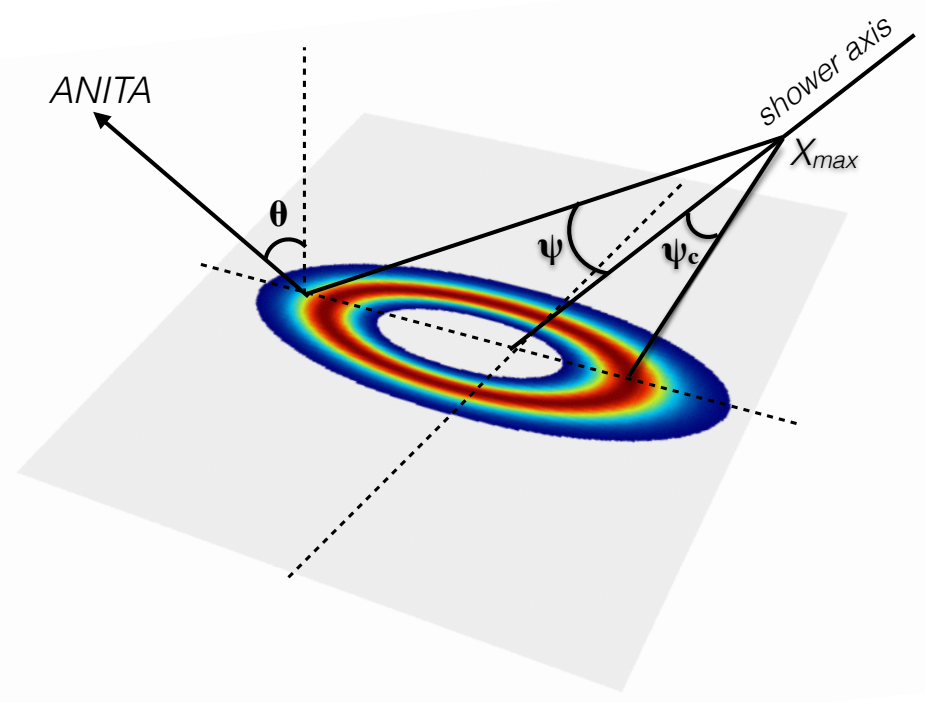

Figure 1: The footprint of the radiation on the ground at a frequency of $300 \mathrm{MHz}$ for an incoming cosmic ray with a zenith angle of $70^{\circ}$ using simulation code from [6]. The atmospheric refractive index causes the coherent radiation to form a Cherenkov-ring. To describe the geometry we use an off-axis angle $\psi$ and the incident angle of the radiation on the surface $\theta$. The off-axis angle of the Cherenkov ring is indicated by $\psi_{c}$.

In this contribution, we develop a method to estimate the energy of UHECRs based on simula- 

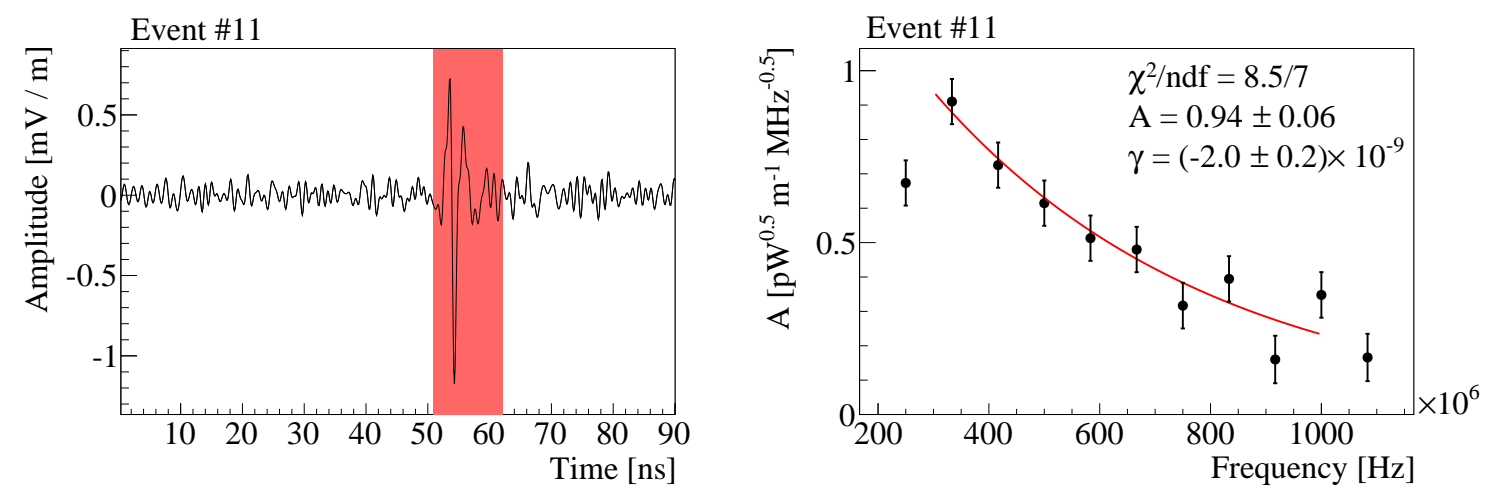

Figure 2: Left: The time dependent electric field induced by an air shower at the location of the ANITA payload. Right: The background corrected amplitude spectrum of the signal window fitted by a simple exponential function.

tions for radio emission from air showers and apply this to the 14 down-going cosmic rays observed. In addition, the full flight is simulated to calculate the exposure and derive the cosmic-ray flux. For a more complete and detailed record of this analysis we refer the reader to [7].

\section{Cosmic-ray energy estimation}

Figure 2 shows an example of the reconstructed electric field at the payload in both the time and frequency domain. As indicated, the impulsive signal is fully contained within a window of about $10 \mathrm{~ns}$. The samples in this window are used to obtain the amplitude spectrum as shown in the right panel of Figure 2. The amplitude spectrum below $300 \mathrm{MHz}$ suffers from loss of power due to filtering of continuous wave sources and inaccuracies in the antenna model. Around $1000 \mathrm{MHz}$, the sensitivity of the signal chain drops rapidly, which makes it impossible to recover any significant signal above this frequency. Therefore, we choose to analyze the amplitude spectrum $A_{f}$ for frequencies $\mathrm{f}$ between 300 and $1000 \mathrm{MHz}$ by fitting a simple exponential function

$$
A_{f}(f)=A e^{\gamma(f-300 \mathrm{MHz})} .
$$

Parameter $A$ gives the amplitude of the electric field at $300 \mathrm{MHz}$ and $\gamma$ describes the frequency dependence of the amplitude spectrum. The motivation to use an exponential function is because it was shown to describe both the amplitude spectrum of simulations [4] and data well.

To calculate the electric field at the payload predicted by simulations, we used an extended version of the ZHAireS code that can handle reflection on a surface [8]. The top panels of Figure 3 shows the behavior of $A$ and $\gamma$ as a function of off-axis angle $\psi$ obtained from a simulation of reflected radiation from an air shower. At the Cherenkov cone the radiation is maximally coherent, resulting in a maximum amplitude and the flattest frequency spectrum. This correlated behavior of $A$ and $\gamma$ as a function of $\psi$ will be exploited in reconstructing the energy of the cosmic ray from the observed radio signal. The bottom left panel of Figure 3 shows, near the Cherenkov cone, the correlation between $A$ and $\psi$. It is well described by a simple linear relationship, $\log _{10}(A)=$ $\log _{10}\left(A_{c}\right)+b\left(\gamma-\gamma_{c}\right)$ and the slope $b$ of this relationship is independent of cosmic-ray energy. This 

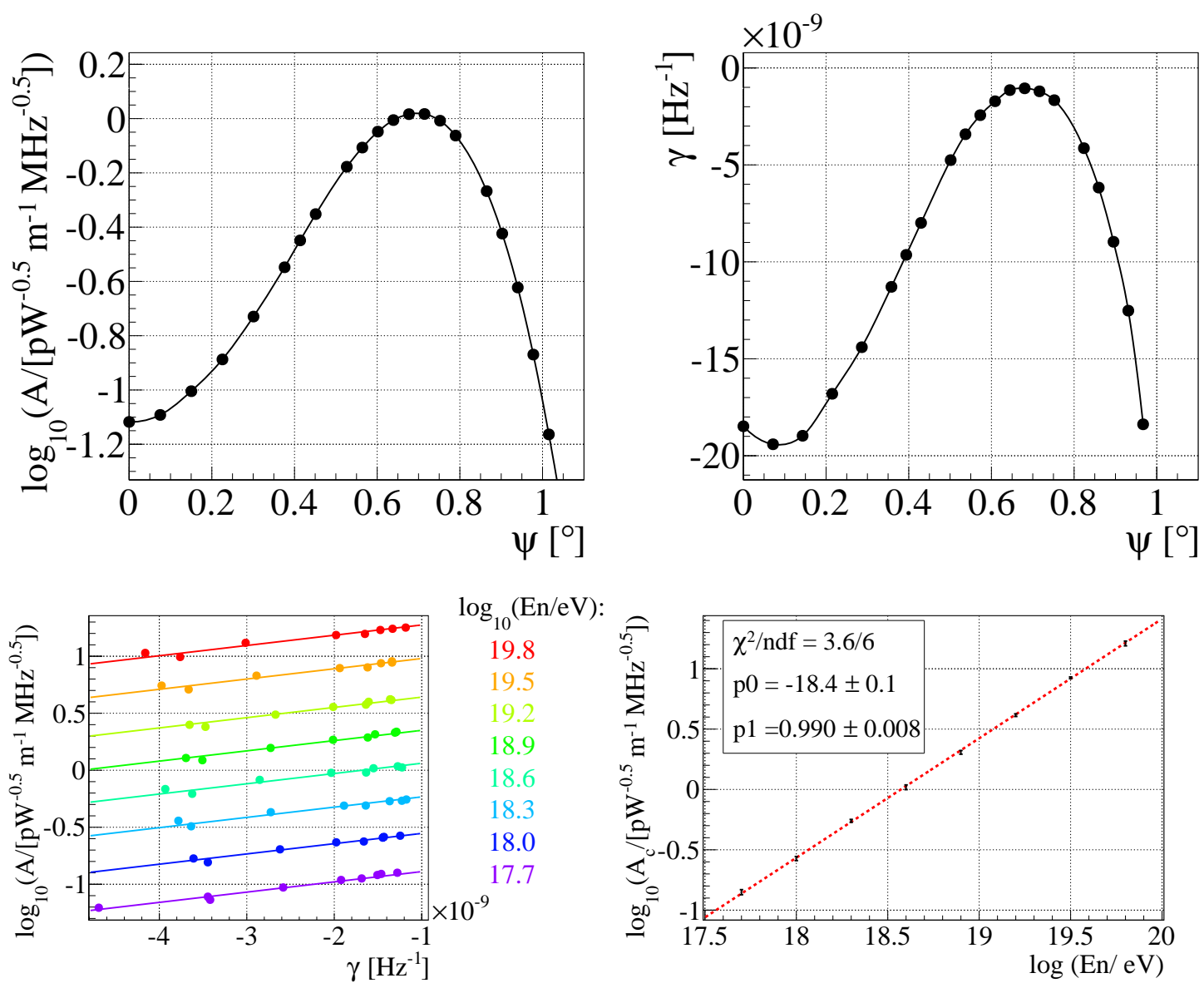

Figure 3: Simulations for energy reconstruction of a cosmic ray with a $70.5^{\circ}$ incident angle. Top Left: The amplitude, $A$, as a function of the off-axis angle, $\psi$ (cosmic-ray energy of $\left.10^{18.6} \mathrm{eV}\right)$. Top Right: The spectral slope $\gamma$ as a function of $\psi$ (cosmic-ray energy of $10^{18.6} \mathrm{eV}$ ). Bottom Left: The correlation between $A$ and $\gamma$ for off-axis angles near the Cherenkov cone for air shower simulations with several cosmic ray energies. To describe these correlations we fit linear functions $\log _{10}(A)=\log _{10}\left(A_{c}\right)+b\left(\gamma-\gamma_{c}\right)$. Bottom Right: Parameters $b$ and $\gamma_{C}$ (obtained from bottom left panel) are used to calculate for a given value $A$ and $\gamma$ the amplitude on the Cherenkov cone $A_{c}$. Each marker represents the mean (and spread) of the $A_{c}$ values obtained from each point in the Figure on the bottom left. By fitting the behavior as function of cosmic ray energy with a simple linear function an energy calibration curve is obtained as indicated by the red dotted line.

means that if we determine $b$ and $\gamma_{c}$ from simulations, we can derive for each measured spectral slope $\gamma$ and amplitude $A$ the corresponding amplitude on the Cherenkov cone $A_{c}$. As shown in bottom right panel of Figure 3, the value of $A_{c}$ is linearly proportional to the cosmic-ray energy. From simulations a cosmic-ray energy calibration curve can be obtained by fitting a first order polynomial $\log _{10} A_{c}=p_{0}+p_{1} \log _{10}\left(E_{C R}\right)$ to the $A_{c}$ values as a function of cosmic-ray energy $E_{C R}$.

There are many factors that influence the amplitude and spectral slope and need to be taken into account for the cosmic-ray energy estimation. In our analysis we address the following:

Reflection coefficients: Local surface roughness and its variation, variation of dielectric properties 


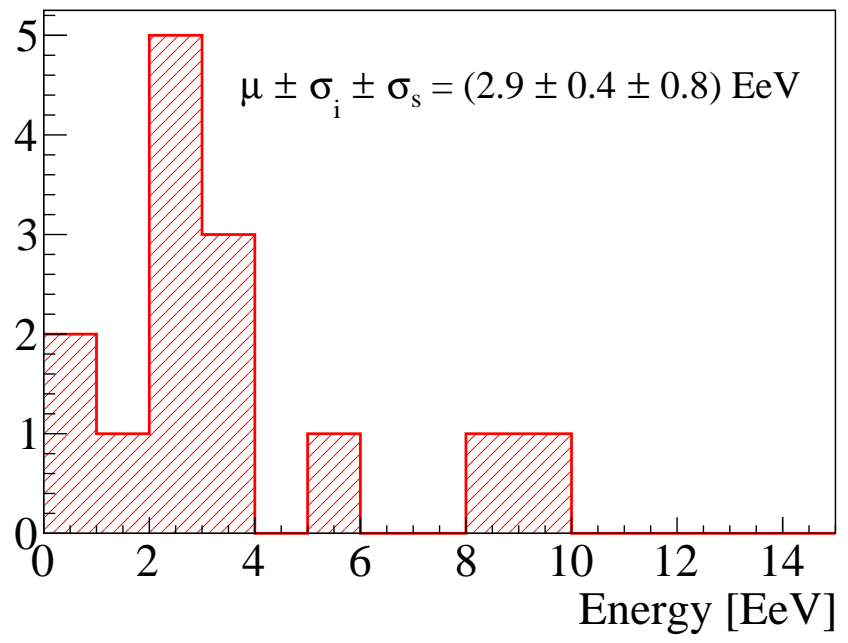

Figure 4: Energy distribution of the 14 cosmic-ray events. Shown are the weighted mean $\mu$, the uncertainties on it due to stochastic uncertainties on the individual events $\sigma_{i}$ and uncertainty on it due to systematic uncertainties on the absolute scale $\sigma_{s}$. The quoted uncertainties represent $95 \%$ confidence intervals.

of the ice, curvature of the Earth.

Atmosphere and Shower Development: Dependence and variation of the depth of shower maximum $\left(X_{\max }\right)$ as a function of energy. Variation in dielectric properties of the atmosphere.

Calibration: Uncertainty and variation in absolute gain of the detector. Uncertainty on the absolute scale of the simulations. Uncertainty on the calibration constants obtained from simulations.

Measurement uncertainties Uncertainty and ambiguity on air shower direction. Noise from background radiation.

After taking into account these factors, the cosmic-ray energy is estimated per event and the distribution is shown in Figure 4. The contributions to the uncertainty that are expected to cause random event-to-event fluctuations in the energy estimate varies between $25 \%$ and $55 \%$. These are used to calculate a weighted mean energy of the cosmic-ray sample which has a value of $2.9 \mathrm{EeV}$. On the mean energy we estimate a $95 \%$ confidence interval $\sigma_{i}$ due to random event-to-event fluctuations of $0.4 \mathrm{EeV}$. In addition to this uncertainty, we estimate an $95 \%$ confidence interval $\sigma_{s}$ due to sources of uncertainty that systematically influence the overall energy scale to be $0.8 \mathrm{EeV}$.

\section{Exposure and cosmic-ray flux calculation}

To calculate ANITA-I flight's exposure, we estimate the detector efficiency as a function of cosmic-ray energy for a simulated isotropic cosmic-ray flux. We simulated several scenarios with different properties of the reflecting surface to estimate the uncertainty on the calculated exposure. 

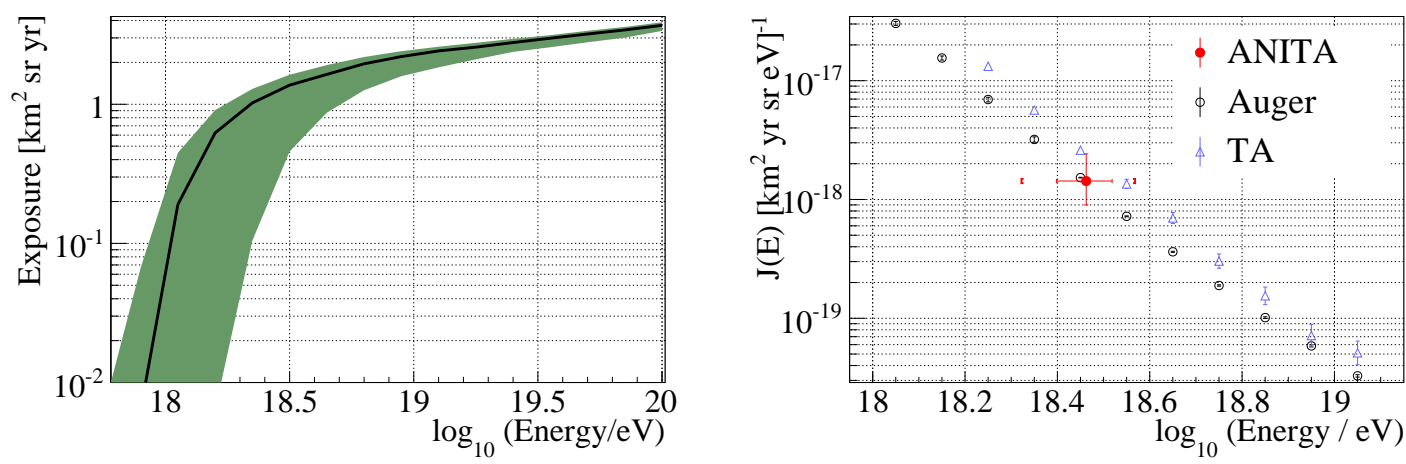

Figure 5: Top: ANITA-I's exposure to UHECRs, derived from Monte Carlo simulations. Bottom: Comparison between the UHECR flux as observed by ANITA-I (see text for details), the Pierre Auger Observatory [9] and the Telescope Array [10].

In the left panel in Figure 5 the black line shows the most appropriate scenario and the green shaded area indicates the region that contains the exposures while variating the surface properties within reasonable limits.

Towards an energy $10^{18.5} \mathrm{eV}$ the exposure grows rapidly indicating that events start to pass the detection threshold. At higher energies the exposure keeps on growing because events generate enough signal further away from their Cherenkov angle to be detected.

In the right panel of Figure 5 the cosmic-ray energy flux $J(E)$ as observed by the Pierre Auger Observatory and the Telescope Array is compared to the flux observed by ANITA-I. Due to the small number of events measured by ANITA and the uncertainty on the exposure, we decided to calculate the flux using a single bin that contains all the observations and use the average reconstructed energy to set the energy scale. The vertical error bar is the combination of the Poisson uncertainty on the number of events and uncertainty on the exposure as indicated by the green band in the left panel of Figure 5.

\section{Conclusion and Outlook}

In this contribution, a new method of cosmic-ray energy reconstruction is presented using observations in the radio frequency range of 300-1000 MHz. Applied to observations made by ANITA-I resulted in a mean energy of $2.9 \pm 0.4\left(\sigma_{i}\right) \pm 0.8\left(\sigma_{s}\right) \times 10^{18} \mathrm{eV}$.

Using a simulation of the full ANITA flight we calculated the exposure for reflected cosmic ray signals and showed that the observed cosmic-ray flux is comparable to observations made elsewhere.

The small cosmic-ray sample observed during the first ANITA-flight and the limited knowledge of the reflection properties of the Antarctic ice limit the accuracy of cosmic-ray energy and flux measurements. In the Austral summer of 2014-2015 the third ANITA flight flew with a significant lower detection threshold. In addition, a companion payload was launched equipped with a pulser in order to probe the reflective properties of the ice surface. Currently several experiments are in place to validate the emission models. For example, the LOFAR [11] and AERA [12] exper- 
iments make accurate observations of radiation from air shower while the T-510 experiment [13] observes radiation from particle beam. It is expected that the combination of all these efforts lead to significant improvement of cosmic-ray flux and energy measurements for the ANITA-III and ANITA-IV missions.

The energy range of the cosmic-ray sample is significantly below the Greisen-Zatsepin-Kuzmin limit and the exposure of balloon based observatories is too small to result in a significant contribution to the total number of UHECR observations. However, the method illustrated in this contribution shows a straight forward cosmic-ray energy estimation while observing the radiation of the air shower at single location. Augmenting existing cosmic-ray observatories with broadband antennas in 300-1000 MHz domain may lead to accurate energy and shower max observations with only a few antennas needed per air shower.

\section{Acknowledgements}

We would like to thank Marianne Ludwig from Karlsruhe Institute of Technology for helpful discussions. We are grateful to NASA, the U.S. National Science Foundation, the U.S. Department of Energy, and the Columbia Scientific Balloon Facility for their generous support of these efforts. We would like to extend our thanks to the 2006-2007 on-ice LDB and McMurdo crews for their support. Part of the research was carried out at the Jet Propulsion Laboratory, California Institute of Technology, under a contract with the National Aeronautics and Space Administration. J. AM, W.R.C., D.G-F and E.Z. thank Ministerio de Economía (FPA2012-39489), Consolider-Ingenio 2010 CPAN Programme (CSD2007-00042), Xunta de Galicia (GRC2013-024), Feder Fundsand Marie Curie-IRSES/ EPLANET (European Particle physics Latin American NETwork), $7^{\text {th }}$ Framework Program (PIRSES- 2009-GA-246806).

\section{References}

[1] P.W. Gorham et al, The Antarctic Impulsive Transient Antenna ultra-high energy neutrino detector: Design, performance, and sensitivity for the 2006-2007 balloon flight, Astroparticle Physics, Volume 32, Issue 1, August 2009, Pages 10-41, ISSN 0927-6505,

[2] S. Hoover et al, Observation of Ultrahigh-Energy Cosmic Rays with the ANITA Balloon-Borne Radio Interferometer, PRL 105, (2010)

[3] K. D. de Vries, A. M. van den Berg, O. Scholten, and K. Werner, Coherent Cherenkov Radiation from Cosmic-Ray-Induced Air Showers, Phys. Rev. Lett. 107, 061101 Published 2 August 2011

[4] J. Alvarez-Muñiz, W. R. Carvalho Jr., A. Romero-Wolf, M. Tueros, E. Zas , Coherent Radiation from Extensive Air Showers in the Ultra-High Frequency Band, Phys. Rev. D86, 123007, 2012

[5] T. Huege, M. Ludwig, C. W. James, Simulating radio emission from air showers with CoREAS, (2013) arXiv:1301.2132

[6] J. Alvarez-Muñiz, W. R. Carvalho Jr., H. Schoorlemmer, E. Zas, Radio pulses from ultra-high energy atmospheric showers as the superposition of Askaryan and geomagnetic mechanisms, Astroparticle Physics, Volume 59, July-August 2014, Pages 29-38, ISSN 0927-6505. 
[7] H. Schoorlemmer et al (The ANITA colaboration), Energy and Flux Measurements of Ultra-High Energy Cosmic Rays Observed During the First ANITA Flight, arXiv:1506.05396, Submitted to Astroparticle Physics.

[8] J. Alvarez-Muñiz, W. R. Carvalho Jr., D. García-Fernández, H. Schoorlemmer, E. Zas. Simulations of reflected radio signals from cosmic ray induced air showers, Astroparticle Physics 66 (2015) 31-38. See also D. García-Fernández et al in these proceedings.

[9] The Pierre Auger Collaboration, The Pierre Auger Observatory: Contributions to the 33rd International Cosmic Ray Conference (ICRC 2013), (2013) arXiv:1307.5059

[10] T. Abu-Zayyad et al (Telescope Array Collaboration), Energy spectrum of ultra-high energy cosmic rays observed with the Telescope Array using a hybrid technique,Astroparticle. Physics. 61, 93 (2015).

[11] A. Nelles, S. Buitink, H. Falcke, J. R. Hörandel, T. Huege, P. Schellart,A parameterization for the radio emission of air showers as predicted by CoREAS simulations and applied to LOFAR measurements, Astroparticle Physics, Volume 60, January 2015, Pages 13-24, ISSN 0927-650

[12] A. Aab et al. (Pierre Auger Collaboration),Probing the radio emission from air showers with polarisation measurements Phys. Rev. D 89, 052002, Published 14 March 2014

[13] See K. Belov et al in these proceedings. 